\title{
Palatal perforation with McGrath Series 5 videolaryngoscope
}

\author{
Semih Arici $\cdot$ Serkan Dogru $\cdot$ Serkan Karaman
}

Received: 12 August 2013/Accepted: 30 August 2013/Published online: 28 September 2013

(C) Japanese Society of Anesthesiologists 2013

Keywords Palate $\cdot$ Soft $\cdot$ Laryngoscopy . Complications

To the Editor:

A healthy pregnant patient (height $166 \mathrm{~cm}$, weight $80 \mathrm{~kg}$ ) who had an adequate preoperative fasting period was taken into the operating room to undergo an elective cesarean section. Induction of anesthesia was provided with thiopental sodium $5 \mathrm{mg} / \mathrm{kg}$ intravenously (IV) and rocuronium bromide $0.6 \mathrm{mg} / \mathrm{kg} \mathrm{IV}$.

Direct laryngoscopy using a Macintosh 3 blade provided a Cormack-Lehane (CL) grade 3 glottic view. Tracheal intubation failed even when the head and neck were repositioned. A McGrath Series 5 videolaryngoscope (MGS-5; Aircraft Medical Limited, Edinburgh, UK) was used to see the glottis. However, no view was obtained from the first attempt. Nevertheless, intubation was successful during the second attempt, under a CL 2 view, with a styletted $7.5-\mathrm{mm}$ endotracheal tube bent $60^{\circ}$ upward just proximal to the cuff according to the angle of the blade. The tip of stylet did not protrude beyond the tube. After removing the MGS-5 from the mouth, blood was seen on the back side of the blade. A direct view of the oral cavity revealed bleeding on the right side of the soft palate. Perforation of the right palate was found, which was repaired by an otorhinolaryngologist during the cesarean section

Electronic supplementary material The online version of this article (doi:10.1007/s00540-013-1709-2) contains supplementary material, which is available to authorized users.

S. Arici $(\bowtie) \cdot$ S. Dogru $\cdot$ S. Karaman

Department of Anesthesiology and Reanimation, School of Medicine, Gaziosmanpasa University, 60100 Tokat, Turkey e-mail: semiharici@gmail.com
(Supplementary Fig. 1). The patient recovered completely. The exact cause why the tube or the blade of MGS-5 caused right palate perforation was not clearly identified.

The MGS-5 is a portable device that provides a better glottic view compared to conventional laryngoscopy and is an alternative to difficult or failed direct laryngoscopy [1, 2]. Besides the existence of palatal injuries using a GlideScope videolaryngoscope, there has been only one case report indicating palatal perforation associated with MGS-5. In their report, the authors concluded that injury occured during tube placement within the blind period of intubation process [1].

This case demonstrates that keeping direct visual contact with the blade of MGS-5 and the tip of tube while inserting it into the oral cavity may minimize the risk of palatal injury.

Conflict of interest None.

\section{References}

1. Williams D, Ball DR. Palatal perforation associated with McGrath videolaryngoscope. Anaesthesia. 2009;64:1144-5.

2. Hirabayashi Y, Seo N. Airway Scope: early clinical experience in 405 patients. J Anesth. 2008;22:81-5. 